\title{
MAXIMIZING NETWORK CAPACITY AND RELIABLE TRANSMISSION IN MIMO COOPERATIVE NETWORKS
}

\author{
K.Swathi ${ }^{1}$, R.V.Krishnaiah ${ }^{2}$ \\ ${ }^{I}$ M.Tech Student Dept. of CS, ${ }^{2}$ Prinicipal, DRKCET, Hyderabad, AP, India \\ kothuriswathi@gmail.com,r.v.krishnaiah@gmail.com
}

\begin{abstract}
Network capacity is an important factor to measure the performance of a network. Cooperative networking is a phenomenon which helps network to have significant gains in terms of transmission reliability and network capacity. Cooperating networking has been applied to multi-hop ad hoc networks. However, the existing works have two limitations. They support only single antenna model and three node relay scheme. The reason behind this limitation due to lack of complete understands of optimal power allocation structure Multiple Input and Multiple Output (MIMO) cooperative networks. Recently Liu et al. studied structural properties with respect to MIMO cooperative networks in presence of node power constraints. Each power allocation at source follows corresponding MIMO structure so as to ensure optimal power allocation. They establish relationship between cooperative relay and pure relay to quantify performance gain. In this paper we did experiments on this concept. Our simulations reveal that the proposed system for cooperative network is able to achieve both transmission reliability and network capacity.
\end{abstract}

Keywords -Cooperative networking, MIMO, optimal power allocation, transmission reliability

\section{INTRODUCTION}

Cooperative networking is said to have capabilities to improve network performance and provide transmission reliability. It has been around since 1970s [1]. [2]. Later on "relay channels" concept came into existence with information theoretic studied [3], [4]. Of late cooperative networking has got more popularity in research circles. Many problems have been studied to solve with cooperative networking concept. They include MAC layer protocol design [5], distributed routing [6], network lifetime maximization [7], and throughput-optimal scheduling [8]. The studied on cooperative networking have limitations. They include optimization is limited to three-node relay scheme, and single antenna systems. Figure 1 shows basic three-node relay scheme.

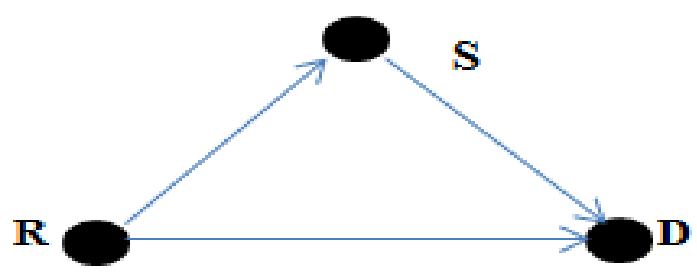

Fig. 1 -Basic three-node relay scheme

As can be seen in figure 1, a message is transmitted from source to destination. However, it is relayed by node $\mathrm{R}$ as it can overhear the message. In case of multi-hop ad hoc network, the message sent from source is likely to be overheard by multiple relay nodes. In this scenario a cooperative networking approach chooses only one neighboring node to act as relay node [9], [10]. Though it is a simple phenomenon, it is suboptimal in performance. Another problem with cooperative network is that it is limited to single - antenna systems. Figure 2 illustrates this scenario.

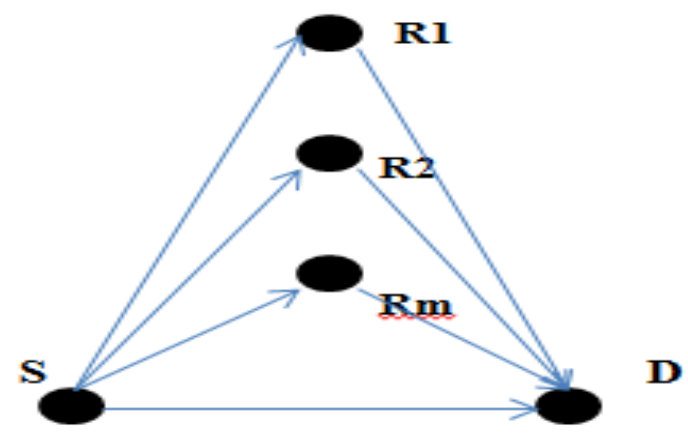

Fig. 2 -Cooperative network with multiple relays.

As can be seen in figure 2, multiple relays are there between the source and destination. The ideal of deploying multiple antennas at each node is very interesting. Multiplexing independent data stream is possible with multiple antennas. Independent spatial channels are exploited in order to achieve this. The two problems found in traditional cooperative networks are somehow related. To find the relation between 
them a single-antenna multi-relay network is considered as shown in figure 2. Analyzing multiple relay networks is somehow related to analyzing three-node relay network. In this paper we focused on the optimal power allocation at source in such a way that it maximizes network capacity and improves reliability of transmission. Our relay strategy is "amplify-and-forward (AF)". This is because there is less complexity in AF strategy. Moreover it is an attractive solution in MIMO cooperative networks. Another important reason to use $\mathrm{AF}$ is that it can outperform DF (decode-andforward) strategy in terms of network capacity. For SNR also $\mathrm{AF}$ is better when compared to DF [11].

In this paper our contribution to implement a cooperative network that demonstrates the proof of concept that improves network capacity besides improving transmission reliability. The remainder of the paper is structured as follows. Section II provides review of literature on cooperative networks. Section III provides information about the proposed work. Section IV presents experimental results while section $\mathrm{V}$ concludes the paper.

\section{RELATED WORKS}

Networking has become ubiquitous for computing. There are Cooperative networks have been around for many years. They are meant for improving the network capacity and also transmission reliability. After initial study on cooperative networks, many works came into existence to improve it further [12], [13], [14], [15], [16], and [17]. In [17] optimal relay amplification matrix is used for improving performance in three-node cooperative network with multiple inputs and multiple outputs. In [16] also different technique of same kind is used. Generalization of matching results to a MIMO-CN with three node network is done in [18]. More complex relay settings were explored of late. Multiple AF relays were studied in [19]. There work is different from the work done by us in this paper as they assumed power constraints at all relay nodes in the network. This approach is not realistic. To overcome this problem we proposed per node power constraints to make it more appropriate. More challenging power allocation problem is thus encountered here. The three node concept given in [13] can also be used with multiple relays. The main different between our work and that of [20] is that there is no cooperation concept in [20] while the node sin our approach cooperate.

\section{PROPOSED COOPERATIVE NETOWRK}

\section{SYSTEM}

The proposed cooperative networking concept is meant for maximizing network capacity. It also contributes to the reliable transmission of data.
Figure 3 shows AF-based MIMO cooperative network.

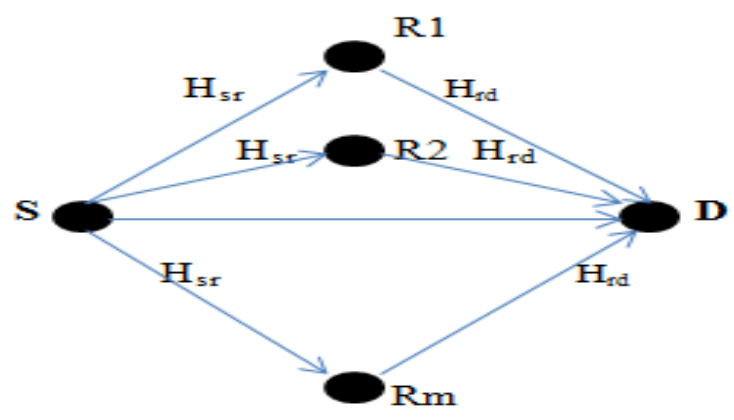

Fig. 3 - AF based MIMO Cooperative network

As can be seen in figure 3, the direct link between source and destination is known as pure relay. If there are other nodes involved in the relay we call it cooperative relay. The source node $\mathrm{S}$ sends message which is supposed to reach destination. The transmission of the data is coordinated by many relay nodes such as R1, R2, .., RM. The antennas of source $\mathrm{S}$ and destination D have antennas Ns and Nd respectively. We also assume that $\mathrm{Ri}$ has $\mathrm{Nr}$ antennas. More technical details of the approach can be found in [21].

\section{OPTIMAL POWER ALLOCATION STRUCTURE}

The cooperative relay case is explored here. When there is direct link between source and destination, it is known as pure relay. When there is no direct link between source and destination and communication takes place through intermediate relay nodes, it is known as cooperative relay. The intermediate nodes in ad hoc networks overhear the communication between the source and destination. Under cooperative relay (CR) we studied the optimal structures of A and Q. As explored priori in [12] Q is fixed. When compared with pure relay finding the optimal structure. The goal of this paper is to find optimal A and Q in order to maximize ICR (Q, A) under CR. It is expressed as follows.

$$
\begin{array}{ll}
\text { Maximize } & I_{C R}(\mathrm{Q}, \mathrm{A}) \\
\text { subject to } & \mathrm{Q} \in \Omega, \mathrm{A} \in \Psi
\end{array}
$$

Further mode the following shows the achievable rate expression.

$$
\begin{aligned}
& I_{P R}(\mathrm{Q}, \mathrm{A})=\frac{1}{2} \log _{2} \mid \mathrm{I}_{M N_{r}}+ \\
& \quad \tilde{\Lambda}_{s r}\left(\mathbf{I}_{M N_{r}}-\left(\mathrm{I}_{M N_{r}}+\tilde{\mathrm{U}}_{s r}^{\dagger} \tilde{\mathrm{H}}_{r d}^{\dagger} \tilde{\mathrm{H}}_{r d} \tilde{\mathrm{U}}_{s r}\right)^{-1}\right) .
\end{aligned}
$$


For given $\mathrm{Q}$, the best relay amplification matrix is as shown below.

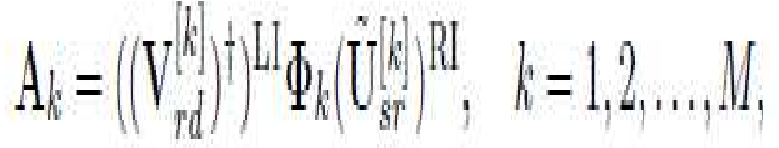

The total gain of the network with respect to network capacity is computed as follows.

$$
\begin{aligned}
& I_{C R}(\mathbf{Q}, \mathbf{A})=\underbrace{\frac{1}{2} \log _{2}\left|\mathbf{I}_{N_{d}}+\frac{1}{\sigma_{d}^{2}} \mathbf{H}_{s d} \mathbf{Q} \mathbf{H}_{s d}^{\dagger}\right|}_{\text {Direct link without relays }}+ \\
& \underbrace{\frac{1}{2} \log _{2}\left|\mathbf{I}_{N_{d}}+\overline{\mathbf{H}}_{s d}\left(\mathbf{Q}^{-1}+\frac{1}{\sigma_{s d}^{2}} \mathbf{H}_{s d}^{\dagger} \mathbf{H}_{s d}\right)^{-1} \overline{\mathbf{H}}_{s d}^{\dagger} \overline{\mathbf{R}}^{-1}\right|}_{\text {Gain from AF relays }}
\end{aligned}
$$

Once $\mathrm{Q}$ and $\mathrm{A}$ are decomposed, the next step is to optimize them. As per the PO-PR for ICR, where Q value is fixed and readily solved. Therefore the rest of the process is focused here. Towards global optimization approach, an algorithm is

\begin{tabular}{|c|}
\hline$g_{01}$ \\
\hline 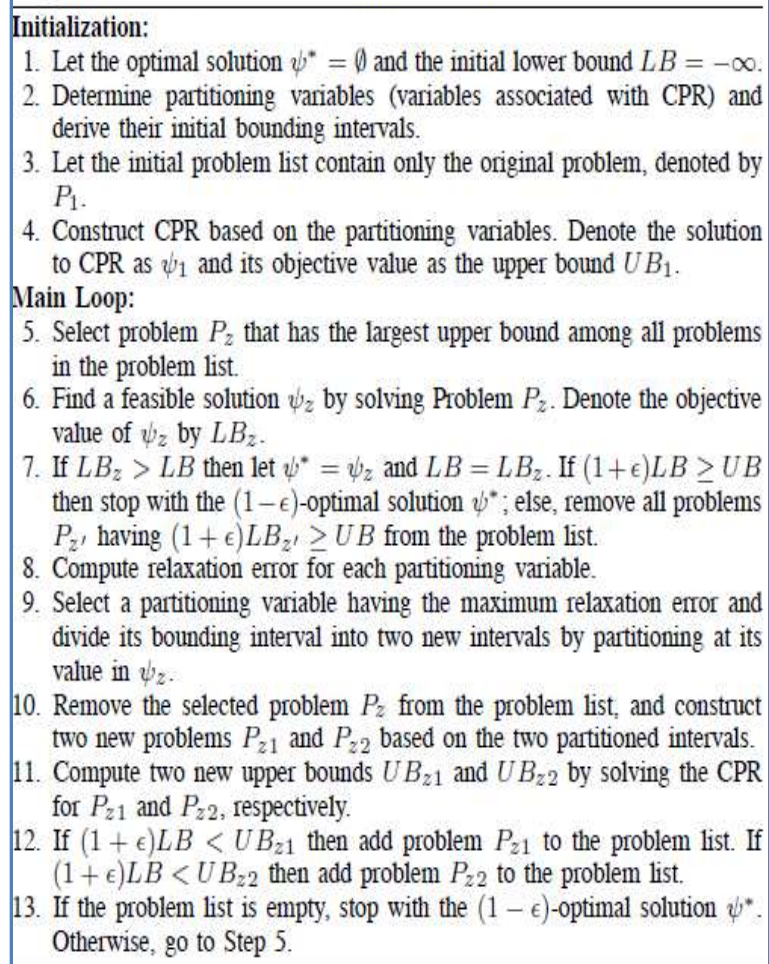 \\
\hline
\end{tabular}
proposed to optimize the A and Q. The algorithm is as shown in figure 4.

Fig. 4 -Algorithm to optimize solution (excerpt from [22])
As can be seen in figure 4, it is evident that the optimal solution is achieved through the procedure given. The optimization process is iterative in nature. More technical details of the algorithm can be found in [22].

\section{RESULTS}

We built a prototype application to simulate the proof of concept. The application demonstrates the concept of cooperative networking. The environment used to build the application is a PC with 4 GB RAM, core 2 dual processor running Windows 7 operating system. The simulations are made through the application. The experiments are carried out in terms of

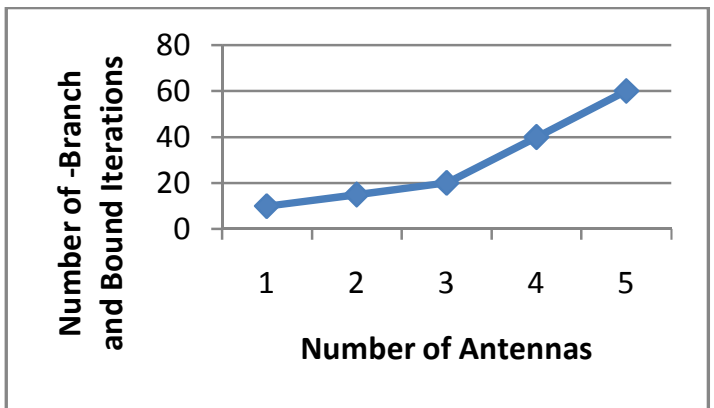

Fig. 5 -Number of iterations vs. antennas

As can be seen in figure 5, the number of antennas is represented by horizontal axis while the number of iterations is represented by vertical axis. Ten randomly generated network instances are averaged to represent each data point. Based on the number of per-node antennas, the iterations are changed proportionately.

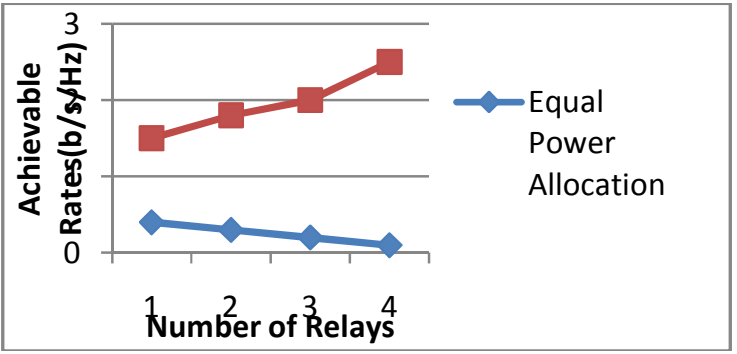

Fig. 6-Number of relays vs. achievable rates (relay changes from 2 to 5 )

As can be seen in figure 6, the number of relays is represented by horizontal axis while the achievable rates are represented by vertical axis. Under optimal power allocation, the achievable rates are compared. The results revealed that there is significant increase in rate gain under optimal power allocation. It does mean that with optimal power allocation, 
when number of relays increase, there is increase in rate gains as well.

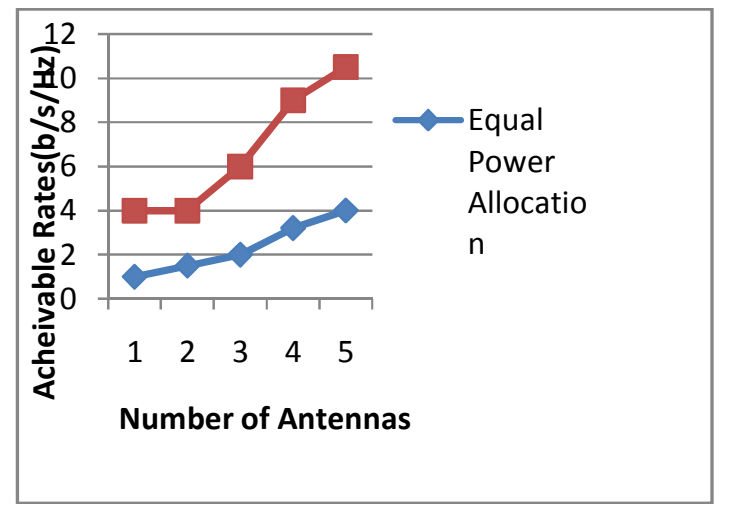

Fig. 7 - Number of relays vs. achievable rates (antenna changes from 2 to 6 )

As can be seen in figure 7, the number of relays is represented by horizontal axis while the achievable rates are represented by vertical axis. Under optimal power allocation, the achievable rates are compared. The results revealed that there is significant increase in rate gain under optimal power allocation. It does mean that with optimal power allocation, when number of relays increase, there is increase in rate gains as well.

\section{CONCLUSIONS}

Structural properties of MIMO cooperative network can have their impact on the network capacity and reliable transmission. In this paper we studied these properties in order to improve network capacity and transmission reliability. Especially we focused on the optimal power allocation in multi-relay MIMO cooperative networks. We implemented algorithms to achieve this. We generalized the settings of three - node relay to multi-relay setting with per node constraints. They we quantified the performance gains to find the relationship between pure relay and cooperative relay. We built simulations to demonstrate the proof of concept. The empirical results revealed that the proposed algorithms are effective in maximizing network capacity with reliable transmission.

\section{REFERENCES}

[1] A. Sendonaris, E. Erkip, and B. Aazhang, "User cooperation diversity- Part I and Part II," IEEE Trans. Commun., vol. 51, no. 11, pp. 1927-1948, Nov. 2003.

[2] J. N. Laneman, D. N. Tse, and G. W. Wornell, "Cooperative diversityin wireless networks: Efficient protocols and outage behavior," IEEETrans. Inf. Theory, vol. 50, no. 12, pp. 3062-3080, Dec. 2004.
[3] T. M. Cover and A. A. El Gamal, "Capacity theorems for the relaychannel," IEEE Trans. Inf. Theory, vol. 25, no. 5, pp. 572-584, Sep.1979.

[4] E. C. van der Meulen, "Three-terminal communication channels," AdvancedApplied Probability, vol. 3, pp. 120-154, 1971.

[5] P. Liu, Z. Tao, S. Narayanan, T. Korakis, and S. S. Panwar, "CoopMAC:A cooperative MAC for wireless LANs," IEEE J. Sel. Areas Commun.,vol. 25, no. 2, pp. 340-354, Feb. 2007.

[6] S. Sharma, Y. Shi, Y. T. Hou, H. D. Sherali, and S. Kompella, "Cooperative communications in multi-hop wireless networks: Jointflow routing and relay node assignment," in Proc. IEEE INFOCOM,San Diego, CA, Mar. 15-19, 2010, pp. $1-9$.

[7] T. Himsoon, W. P. Siriwongpairat, Z. Han, and K. J. R. Liu, "Lifetimemaximization via cooperative nodes and relay deployment in wirelessnetworks," IEEE J. Sel. Areas Commun., vol. 25, no. 2, pp. 306-317,Feb. 2007.

[8] E. M. Yeh and R. A. Berry, "Throughput optimal control of cooperativerelay networks," IEEE Trans. Inf. Theory, vol. 53, no. 10, pp. 3827-3833, Oct. 2007.

[9] Y. Shi, S. Sharma, Y. T. Hou, and S. Kompella, "Optimal relayassignment for cooperative communications," in Proc. ACM MobiHoc,Hong Kong SAR, China, May 2008, pp. 312.

[10] J. Cai, X. Shen, J. W. Mark, and A. S. Alfa, "Semidistributed userrelaying algorithm for amplify-and-forward wireless relay networks,'IEEE Trans. Wireless Commun., vol. 7, no. 4, pp. 1348-1357, Apr.2008.

[11] J. Liu, N. B. Shroff, and H. D. Sherali, "Optimal power allocationin multi-relay MIMO cooperative networks: Theory and algorithms,"'Technical Report, Deptment of ECE, Ohio State University, Jul. 2010.[Online]. Available: http://www2.ece.ohio-state.edu/ $\square$ liu/publications/AF MIMO MRELAY TR.pdf

[12] B. Khoshnevis, W. Yu, and R. Adve, "Grassmannianbeamforming forMIMO amplify-and-forward relaying," IEEE J. Sel. Areas Commun.,vol. 26, no. 8, pp. 1397-1407, Oct. 2008.

[13] Y. Rong, X. Tang, and Y. Hua, "A unified framework for optimizing linearnonregenerative multicarrier MIMO relay communication systems,"IEEE Trans. Signal Process., vol. 57, no. 12 , pp. 4837-4851, Dec. 2009.

[14] A. Behbahani, R. Merched, and A. M. Eltawil, "Optimizations of aMIMO relay network," IEEE Trans. Signal Process., vol. 56, no. 10,pp. 5062-5073, Oct. 2008.

[15] W. Guan, H. Luo, and W. Chen, "Linear relaying scheme for MIMOrelay system with QoS requirements," IEEE Signal Process. Lett.,vol. 15, pp. 697-700, 2008.

[16] O. Mũnoz-Medina, J. Vidal, and A. Agust'1n, "Linear transceiver designin nonregenerative relays with channel state information," IEEE Trans.Signal Process., vol. 55, no. 6, pp. 2593-2604, Jun. 2007. 
[17] X. Tang and Y. Hua, "Optimal design of nonregenerative MIMOwireless relays," IEEE Trans. Wireless Commun., vol. 6, no. 4, pp. 1398-1407, Apr. 2007.

[18] Z. Fang, Y. Hua, and J. D. Koshy, "Joint source and relay optimizationfor non-regenerative MIMO relay," in Proc. IEEE Workshop SensorArray Multi-Channel Signal Processing, Waltham, WA, Jul. 12-14, 2006,pp. 239-243.

[19] Y. Fu, L. Yang, W.-P. Zhu, and Z. Yang, “A convex optimization design of relay precoderfor two-hop MIMO relay networks," in Proc. IEEEICC, Cape Town, South Africa, May 23-27, 2010.

[20] Y. Rong, "Non-regenerative multicarrier MIMO relay communicationsbased on minimization of mean-squared error," in Proc. IEEE ICC,Dresden, Germany, Jun. 14-18, 2009, pp. 1-5.

[21] Jia Liu, Ness B. ShroffandHanif D. Sherali, "Optimal Power Allocation in Multi-Relay MIMO Cooperative Networks: Theory and Algorithms". IEEE JOURNAL ON SELECTED AREAS IN COMMUNICATIONS, VOL. 30, NO. 2, FEBRUARY 2012

[22] G. L. Nemmhauser and L. A. Wolsey, Integer and CombinatorialOptimization, 2nd ed. New York: WileyInterscience Publication, 1999.

\section{BIOGRAPHIES:}

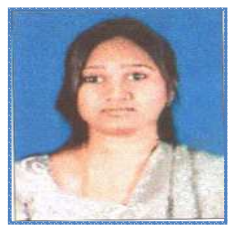

K. Swathi has completed MCA from Nava Bharathi College of PG studies and pursuing M.Tech(C.S) in DRK College of Engineering and Technology, JNTU, Hyderabad, Andhra Pradesh, India. Her main research interest includes Mobile Communications \& Computer Networks.

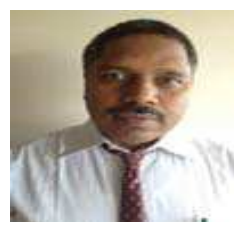

Dr. R. V. Krishnaiah, did M.Tech (EIE) from NIT Waranagal, MTech(CSE) form JNTU, ,Ph.D, from JNTU Ananthapur, He has memberships in professional bodies MIE, MIETE, MISTE. His main research interests include Image Processing, Security systems, Sensors, Intelligent Systems, Computer networks, Data mining, Software Engineering, network protection and security control. He has published many papers and Editorial Member and Reviewer for some national and international journals. 